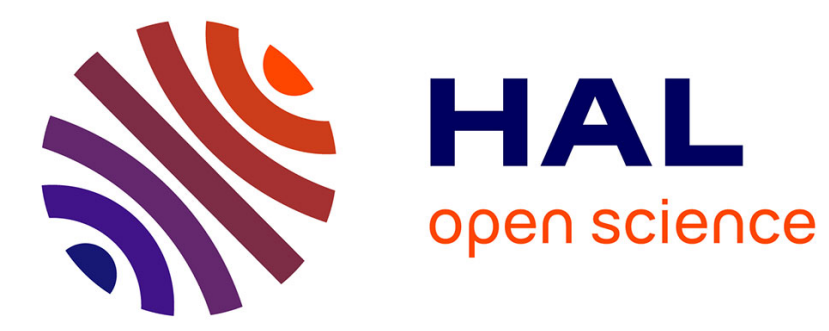

\title{
Utilisation des plasmas pour l'accélération de particules à très haute énergie \\ P. Mora
}

\section{To cite this version:}

P. Mora. Utilisation des plasmas pour l'accélération de particules à très haute énergie. Revue de Physique Appliquée, 1988, 23 (9), pp.1489-1494. 10.1051/rphysap:019880023090148900 . jpa00245975

\section{HAL Id: jpa-00245975 https://hal.science/jpa-00245975}

Submitted on 1 Jan 1988

HAL is a multi-disciplinary open access archive for the deposit and dissemination of scientific research documents, whether they are published or not. The documents may come from teaching and research institutions in France or abroad, or from public or private research centers.
L'archive ouverte pluridisciplinaire HAL, est destinée au dépôt et à la diffusion de documents scientifiques de niveau recherche, publiés ou non, émanant des établissements d'enseignement et de recherche français ou étrangers, des laboratoires publics ou privés. 


\title{
Utilisation des plasmas pour l'accélération de particules à très haute énergie
}

\author{
P. Mora \\ Centre de Physique Théorique et Greco Interaction Laser-Matière, Ecole Polytechnique, 91128 Palaiseau \\ Cedex, France
}

(Reçu le 15 octobre 1987, accepté le 20 mai 1988)

\begin{abstract}
Résumé. - Les plasmas peuvent être le siège de champs électrostatiques de grande amplitude. Si $n_{\mathrm{e}}$ est la densité électronique, si $\delta$ est l'amplitude relative de la perturbation de densité associée à une onde plasma électronique, et si la vitesse de phase de cette onde est proche de la vitesse de la lumière, l'amplitude du champ électrique associé à l'onde est donnée par $E(\mathrm{GV} / \mathrm{m})=10^{3} \delta\left(n_{\mathrm{e}}\left(\mathrm{cm}^{-3}\right) / 10^{20}\right)^{1 / 2}$. On conçoit donc l'intérêt que peuvent représenter les plasmas denses pour la conception de nouveaux accélérateurs de particules. Plusieurs méthodes ont été proposées pour générer de telles ondes dans les plasmas. L'excitation résonnante par battement de deux ondes lasers est un des concepts les plus séduisants. On discute dans cet article des mécanismes de saturation de l'onde, en particulier ceux dus à la nature volontiers instable du plasma.
\end{abstract}

\begin{abstract}
Large amplitude electrostatic fields may develop in plasmas. Let $n_{\mathrm{e}}$ be the electron density, $\delta$ the relative amplitude of the density perturbation due to an electron plasma wave. If the phase velocity of the wave is approximately equal to the light velocity, the amplitude of the electric field of the wave is $E(\mathrm{GV} / \mathrm{m})=10^{3} \delta\left(n_{\mathrm{e}}\left(\mathrm{cm}^{-3}\right) / 10^{20}\right)^{1 / 2}$. One easily sees the interest of plasmas for the conception of future accelerators of particles. Different methods have been proposed to generate such large amplitude waves. The resonant excitation by the beating of two lasers is one of the most attractive. In this paper, we discuss the saturation mechanisms of the wave.
\end{abstract}

\section{Intérêt des plasmas pour les accélérateurs.}

Si nous pouvons être certains que les accélérateurs de particules actuels permettront d'obtenir des informations fondamentales pour étendre nos connaissances dans le cadre du modèle standard, nous savons aussi que ce modèle est incomplet, et que des énergies bien supérieures seront nécessaires pour répondre à des questions fondamentales (origine des masses, des familles de quarks et leptons, nombre de neutrinos, ...), et atteindre une possible unification des forces de la nature.

Depuis une quarantaine d'années, les progrès de la recherche en physique des particules élémentaires ont été dominés par l'augmentation exponentielle dans le temps de l'énergie des accélérateurs de particules, de l'ordre d'un facteur dix tous les sept ans. Cette réussite surprenante est due à l'émergence successive de nouvelles techniques d'accélération.

La taille des accélérateurs circulaires actuels est déterminée par l'intensité du champ magnétique que l'on sait produire, et par les pertes par rayonnement synchrotron, qui augmentent considérablement avec l'énergie des particules. On s'oriente donc maintenant vers les accélérateurs linéaires, qui ne souffrent pas de ces limitations. Des champs accélérateurs de l'ordre de quelques dizaines de $\mathrm{MV} / \mathrm{m}$ sont obtenus dans ces machines, mais il est douteux que l'on puisse dépasser de beaucoup ces valeurs, à cause des problèmes de claquage au voisinage des parois des structures accélératrices. Pourtant, dans le futur, l'objectif à atteindre si l'on veut éviter des dimensions d'accélérateurs gigantesques est plutôt dans la gamme des $\mathrm{GV} / \mathrm{m}$.

Pour atteindre ces valeurs de champs électriques, différentes idées ont été proposées. Plusieurs d'entre elles utilisent un plasma comme milieu accélérateur. Une limitation du champ électrique dans les accélérateurs à base de cavités résonnantes tient, on l'a vu, au claquage des matériaux. Au contraire, un plasma chaud étant un milieu totalement ionisé, cette limitation n'existe pas, et des champs électriques très 
élevés, de l'ordre de 10 à $100 \mathrm{GV} / \mathrm{m}$ peuvent y être obtenus par simple séparation spatiale des charges positives et négatives du plasma. Ces champs de séparation de charge se propagent alors sous forme d'ondes longitudinales, les ondes plasmas électroniques. Si la vitesse de phase de l'onde est proche de la vitesse de la lumière, si $n_{\mathrm{e}}$ est la densité électronique du plasma, et si $\delta n$ est l'amplitude de la perturbation de densité électronique associée à l'onde, l'amplitude du champ électrique est donnée par :

$$
E=\left(e c / \varepsilon_{0} \omega_{\mathrm{p}}\right) \delta n,
$$

où $\omega_{\mathrm{p}}$ est la fréquence plasma électronique, reliée à la densité électronique $n_{\mathrm{e}}$ du plasma par: $\omega_{\mathrm{p}}^{2}=n_{\mathrm{e}} e^{2} / m_{\mathrm{e}} \varepsilon_{0}$, soit

$$
E(\mathrm{GV} / \mathrm{m})=10^{3}\left(n_{\mathrm{e}}\left(\mathrm{cm}^{-3}\right) / 10^{20}\right)^{1 / 2}\left(\delta n / n_{\mathrm{e}}\right) .
$$

Comment exciter ces ondes longitudinales de grande amplitude? Une des méthodes les plus prometteuses, proposée en 1979 par Tajima et Dawson [1], utilise le battement de deux ondes lasers dans les plasmas. Cette méthode cherche à utiliser la très grande densité d'énergie qui se trouve au voisinage du foyer d'un faisceau laser de grande puissance pour générer un champ électrique longitudinal très intense.

Deux ondes lasers de fréquences $\omega_{1}$ et $\omega_{2}$ sont focalisées dans un plasma de fréquence plasma électronique $\omega_{\mathrm{p}}$, telle que $\omega_{1}-\omega_{2}=\omega_{\mathrm{p}}$. Le battement entre les deux ondes excite alors de façon résonnante l'oscillation plasma à la fréquence $\omega_{\mathrm{p}}$. Si $\omega_{1}$ est grand devant $\omega_{\mathrm{p}}$, l'onde plasma ainsi créée a une vitesse de phase proche de la vitesse de la lumière. Des travaux expérimentaux effectués aux Etats-Unis et au Canada ont montré récemment que des ondes de battement, avec des amplitudes supérieures au $\mathrm{GV} / \mathrm{m}$, ont d'ores et déjà été obtenues à l'aide de lasers à gaz carbonique, de longueurs d'onde voisines de $10 \mu \mathrm{m}$ [2].

Il existe différentes variantes de la méthode du battement d'ondes lasers. L'une d'entre elles est la méthode de génération de l'onde plasma électronique par instabilité Raman avant [3], où l'onde plasma électronique et la deuxième onde électromagnétique sont générées simultanément dans le plasma par instabilité paramétrique d'une seule onde laser très intense. La condition de résonance $\omega_{1}-\omega_{2}=\omega_{\mathrm{p}}$ est alors automatiquement réalisée, mais le contrôle de l'onde plasma générée est plus délicat. Une deuxième variante est le battement laser-onduleur, où la deuxième onde laser est remplacée par un champ magnétique spatialement oscillant, en présence d'électrons relativistes [4].

Une autre méthode très séduisante pour exciter des ondes longitudinales de grande amplitude est la méthode de champ de sillage dans les plasmas, lors du passage d'un faisceau primaire, qui peut être un faisceau lumineux (impulsion laser ultra-courte) ou un premier faisceau de particules chargées relativistes. Expérimentalement moins avancée, cette méthode bénéficie cependant d'un rendement théorique plus élevé.

Dans la suite de l'article, on s'intéresse à une onde plasma excitée par le battement de deux ondes lasers. Dans le deuxième paragraphe, on estime les niveaux de saturation atteints par l'onde plasma sous l'effet de divers mécanismes, en supposant l'onde purement monodimensionnelle. Dans le troisième paragraphe, on étudie les effets transverses dus à la dimension finie de la tache focale. Le quatrième paragraphe est consacré à l'étude de la destruction de la cohérence de l'onde sous l'effet de l'instabilité modulationnelle. Enfin, en conclusion, on fait le bilan de l'ensemble des mécanismes étudiés, que l'on résume sur une figure donnant l'amplitude de l'onde plasma attendue en fonction du flux laser incident, pour un ensemble de valeurs typiques des paramètres expérimentaux.

\section{Saturation de l'onde plasma électronique.}

On considère donc deux ondes lasers de fréquences $\omega_{1}$ and $\omega_{2}$ se propageant dans un plasma de densité $n_{\mathrm{e}}$ telle que $\omega_{1}-\omega_{2}=\omega_{\mathrm{p}}$. Soient $E_{1}$ et $E_{2}$ les amplitudes des champs électriques des deux ondes lasers, et $\alpha_{1}$ et $\alpha_{2}$ les quantités correspondantes normalisées, égales au rapport de l'amplitude de la vitesse d'oscillation dans le champ électrique de chacune des deux ondes à la vitesse de la lumière,

$$
\alpha_{1,2}=\frac{e E_{1,2}}{m_{\mathrm{e}} \omega_{1,2} c} .
$$

On définit de même la perturbation de densité relative $a=\delta n / n_{\mathrm{e}}$ (plus précisément son amplitude complexe) associée à l'onde plasma électronique générée par le battement à la fréquence $\omega_{\mathrm{p}}$, encore égale au rapport de la vitesse d'oscillation dans le champ électrique $E_{\mathrm{p}}$ de l'onde plasma à la vitesse de la lumière,

$$
a=\frac{\delta n}{n_{\mathrm{e}}}=\frac{e E_{\mathrm{p}}}{m_{\mathrm{e}} \omega_{\mathrm{p}} c} .
$$

Elle satisfait l'équation d'évolution suivante (équation d'enveloppe) [5],

$$
\begin{aligned}
\frac{1}{\omega_{\mathrm{p}}} \frac{\mathrm{d} a}{\mathrm{~d} t}=\frac{1}{4} \alpha_{1} \alpha_{2} & +\frac{3}{16} i|a|^{2} a- \\
& -i \frac{\omega_{\mathrm{p}}-\left(\omega_{1}-\omega_{2}\right)}{\omega_{\mathrm{p}}} a-\frac{\nu}{2 \omega_{\mathrm{p}}} a .
\end{aligned}
$$

Le premier terme dans le membre de droite correspond à la source provenant du battement des deux ondes lasers. Le second terme est le désaccord de fréquence non linéaire, dû à la variation relativiste de masse de l'électron dans le champ électrique de 
l'onde plasma. Ce terme est obtenu par un développement limité du facteur relativiste en puissance de l'amplitude $a$. Le troisième terme est le désaccord de fréquence linéaire, dû à un ajustement imparfait de la densité électronique à la fréquence du battement. Finalement, le dernier terme correspond à l'amortissement collisionnel de l'onde.

Dans la suite, on donne les expressions analytiques et les valeurs numériques pour les amplitudes maximum atteintes par la perturbation relative de densité $a=\delta n / n_{\mathrm{e}}$ en étudiant séparément chacun des phénomènes pouvant conduire à la saturation de l'onde. Pour les évaluations numériques, on suppose que les deux lasers ont la même densité de flux d'énergie $\Phi$, et deux longueurs d'ondes voisines $\lambda_{1} \approx \lambda_{2} \approx \lambda$. On normalise la densité de flux d'énergie laser, la longueur d'onde laser, la densité et la température du plasma de la façon suivante :

$$
\begin{aligned}
\Phi_{14} & =\Phi\left(\mathrm{W} \mathrm{cm}^{-2}\right) / 10^{14} \\
\lambda & =\lambda(\mu \mathrm{m}) \\
n_{17} & =n_{\mathrm{e}}\left(\mathrm{cm}^{-3}\right) / 10^{17} \\
T & =T(\mathrm{eV}) .
\end{aligned}
$$

Considérons tout d'abord la saturation due au terme non linéaire d'origine relativiste. Au fur et à mesure que l'amplitude de l'onde croît, le désaccord entre la fréquence de battement et la fréquence propre effective de l'oscillateur croît, et cela se traduit par un déphasage progressif entre le terme source et l'oscillation plasma. Quand ce déphasage atteint puis dépasse $\pi / 2$, l'amplitude des oscillations cesse de crô̂tre puis rediminue. L'amplitude maximum conséquence du désaccord relativiste de fréquence est alors

$$
\delta n / n_{\mathrm{e}}=\left(16 \alpha_{1} \alpha_{2} / 3\right)^{1 / 3} .
$$

Numériquement, l'amplitude normalisée d'une onde laser vaut

$$
\alpha=0.86 \times 10^{-2}\left(\Phi_{14} \lambda^{2}\right)^{1 / 2}
$$

si bien que l'équation (7) se lit

$$
\delta n / n_{\mathrm{e}}=7.3 \times 10^{-2}\left(\Phi_{14} \lambda^{2}\right)^{1 / 3} .
$$

Le temps de saturation correspondant est relié à l'amplitude maximum par la relation

$$
\delta n / n_{\mathrm{e}}=0.82\left(\alpha_{1} \alpha_{2} / 4\right) \omega_{\mathrm{p}} t_{\text {sat }}
$$

soit, comme

$$
\omega_{\mathrm{p}}=1.79 \times 10^{13} n_{17}^{1 / 2}
$$

il vient

$$
t(\mathrm{ps})=270 n_{17}^{-1 / 2}\left(\Phi_{14} \lambda^{2}\right)^{-2 / 3} .
$$

Considérons maintenant le terme de saturation linéaire. On néglige le terme relativiste, mais on suppose le milieu imparfaitement accordé, dans la mesure où il est difficile de créer un plasma de densité parfaitement défini. On a alors affaire à un oscillateur harmonique excité à une fréquence proche de sa fréquence propre. Soit $\Delta n / n_{\mathrm{e}}$ le désaccord relatif de densité,

$$
\frac{\Delta n}{n_{\mathrm{e}}}=2 \frac{\omega_{\mathrm{p}}-\left(\omega_{1}-\omega_{2}\right)}{\omega_{\mathrm{p}}} .
$$

L'amplitude maximum conséquence du désaccord linéaire de fréquence est alors

$$
\delta n / n_{\mathrm{e}}=\left(n_{\mathrm{e}} / \Delta n\right) \alpha_{1} \alpha_{2}
$$

soit

$$
\delta n / n_{\mathrm{e}}=7.4 \times 10^{-5} \Phi_{14} \lambda^{2}\left(n_{\mathrm{e}} / \Delta n\right)
$$

et le temps de saturation correspondant est donné par

$$
t=2 \pi \omega_{\mathrm{p}}^{-1}\left(n_{\mathrm{e}} / \Delta n\right)
$$

soit

$$
t(\mathrm{ps})=0.35 n_{17}^{-1 / 2}\left(n_{\mathrm{e}} / \Delta n\right) .
$$

Considérons finalement l'amortissement collisionnel négligé dans les calculs précédents. L'amplitude de saturation atteinte vaut :

$$
\delta n / n_{\mathrm{e}}=\left(\omega_{\mathrm{p}} / 2 \nu\right) \alpha_{1} \alpha_{2} .
$$

La valeur de la fréquence de collision $\nu$ dépend de la valeur relative de la vitesse thermique électronique $v_{\mathrm{t}}=\left(T / m_{\mathrm{e}}\right)^{1 / 2}$ et de la vitesse d'oscillation $v_{\text {osc }}$ dans les champs électriques des ondes lasers et de l'onde plasma électronique (voir l'appendice). Quand $v_{t} \gg v_{\text {osc }}$, le niveau de saturation collisionnel est donné par

$$
\delta n / n_{\mathrm{e}}=5.85 \times 10^{-4} \Phi_{14} \lambda^{2} n_{17}^{-1 / 2} T^{3 / 2} .
$$

Quand $v_{\mathrm{t}}, v_{\text {osc, onde plasma }} \ll v_{\text {osc, laser }}$, il suffit de remplacer $T$ par $T_{\text {osc }}$ (définie dans l'appendice) dans l'équation (19), et

$$
\delta n / n_{\mathrm{e}}=0.9 \times 10^{-2}\left(\Phi_{14} \lambda^{2}\right)^{5 / 2} n_{17}^{-1 / 2} .
$$

Quand $v_{t}, v_{\text {osc, laser }} \ll v_{\text {osc, onde plasma }}$, l'amortissement collisionnel est incapable d'empêcher l'onde de croître indéfiniment, en l'absence d'autre mécanisme de saturation, bien entendu. La condition $T_{\text {osc, onde plasma }}>T, T_{\text {osc, laser }}$ s'écrit aussi :

$\delta n / n_{\mathrm{e}}>3.5 \times 10^{-3} T^{1 / 2}, \quad 0.86 \times 10^{-2}\left(\Phi_{14} \lambda^{2}\right)^{1 / 2}$

\section{Effets latéraux dus à la dimension finie de la tache focale.}

Si le laser a une tache focale de dimension finie, la force pondéromotrice associée au rayonnement laser 
expulse le plasma du canal formé par le rayonnement. L'équation du mouvement du plasma s'écrit

$$
\frac{\mathrm{d} \mathbf{v}}{\mathrm{d} t}=-\frac{Z m_{\mathrm{e}}}{m_{\mathrm{i}}} \nabla \frac{v_{\mathrm{osc}}^{2}}{4}
$$

où $v_{\text {osc }}$ est la vitesse d'oscillation dans le champ électrique de l'onde laser. Si on tient compte maintenant de l'équation de continuité

$$
\frac{\partial n}{\partial t}+\frac{\partial n \mathbf{v}}{\partial \mathbf{r}}=0
$$

on obtient que la densité du plasma décroît progressivement, et cela se traduit par un désaccord de fréquence entre le battement et l'onde plasma électronique. Si $l$ est la largeur à mi-hauteur de la tache focale supposée de forme gaussienne, l'onde plasma sature à

$$
\frac{\delta n}{n_{\mathrm{e}}}=0.44\left(\frac{m_{\mathrm{i}}}{Z m_{\mathrm{e}}}\right)^{1 / 3}\left(\frac{\omega_{\mathrm{p}} l}{c}\right)^{2 / 3} \frac{\alpha_{1} \alpha_{2}}{\left(\alpha_{1}^{2}+\alpha_{2}^{2}\right)^{1 / 3}}
$$

c'est-à-dire

$$
\delta n / n_{\mathrm{e}} \approx 2.5 \times 10^{-2}(l / 100 \mu \mathrm{m})^{2 / 3} n_{17}^{1 / 3}\left(\Phi_{14} \lambda^{2}\right)^{2 / 3} .
$$

D'autre part, la force pondéromotrice latérale associée à l'onde plasma électronique elle-même a les mêmes effets. Par un calcul analogue, où $v_{\text {osc }}$ est maintenant la vitesse d'oscillation dans le champ électrique de l'onde plasma, on obtient

$$
\frac{\delta n}{n_{\mathrm{e}}}=0.82\left(\frac{m_{\mathrm{i}}}{Z m_{\mathrm{e}}}\right)^{1 / 5}\left(\frac{\omega_{\mathrm{p}} l}{c}\right)^{2 / 5}\left(\alpha_{1} \alpha_{2}\right)^{3 / 5}
$$

c'est-à-dire

$$
\delta n / n_{\mathrm{e}}=2.5 \times 10^{-2}(l / 100 \mu \mathrm{m})^{2 / 5} n_{17}^{1 / 5}\left(\Phi_{14} \lambda^{2}\right)^{3 / 5} .
$$

\section{Saturation due à l'instabilité modulationnelle de l'onde plasma.}

En plus des précédents mécanismes de saturation, il faut tenir compte de l'éventualité de différentes instabilités qui peuvent détruire la cohérence de l'onde plasma électronique, avant qu'elle n'ait eu le temps d'atteindre une amplitude suffisamment élevée. En particulier, le couplage non linéaire entre une onde plasma de grande amplitude et les perturbations de densité ionique conduit à l'instabilité modulationnelle de l'onde plasma. Pour une onde plasma de grande longueur d'onde et d'amplitude modérément élevée, cette instabilité a le taux de croissance suivant [6]

$$
\gamma=\left(\frac{1}{6} \frac{Z m_{\mathrm{e}}}{m_{\mathrm{i}}}\right)^{1 / 2} \frac{c}{v_{\mathrm{t}}} \frac{\delta n}{n_{\mathrm{e}}} \omega_{\mathrm{p}} .
$$

Si on suppose un flux laser constant, l'amplitude de l'onde plasma électronique, et donc $\gamma(t)$ qui lui est proportionnel, croissent, linéairement dans le temps, si on néglige les autres mécanismes de saturation. Si on admet que la cohérence de l'onde plasma électronique est détruite quand $\int \gamma(t) \mathrm{d} t=e_{\mathrm{c}}$, on trouve que l'amplitude atteinte au moment où est réalisée cette condition est donnée par

$\frac{\delta n}{n_{\mathrm{e}}}=\left(\frac{e_{\mathrm{c}}}{2}\right)^{1 / 2}\left(\frac{6 m_{\mathrm{i}}}{Z m_{\mathrm{e}}}\right)^{1 / 4}\left(\frac{v_{\mathrm{t}}}{c}\right)^{1 / 2}\left(\alpha_{1} \alpha_{2}\right)^{1 / 2}$.

Compte tenu de la valeur numérique de la vitesse thermique,

$$
v_{\mathrm{t}} / c=1.4 \times 10^{-3} T^{1 / 2}
$$

on obtient,

$\delta n / n_{\mathrm{e}}=0.73 \times 10^{-2}\left(e_{\mathrm{c}} / 10\right)^{1 / 2}\left(\Phi_{14} \lambda^{2}\right)^{1 / 2} T^{1 / 4}$

au bout du temps

$$
t(\mathrm{ps})=22\left(e_{\mathrm{c}} / 10\right)^{1 / 2}\left(\Phi_{14} \lambda^{2}\right)^{-1 / 2} n_{17}^{-1 / 2} T^{1 / 4} .
$$

Pour une impulsion laser profilée dans le temps, $\Phi \propto t^{m}$, les formules sont un peu plus compliquées, mais le produit de l'amplitude de saturation par le temps de saturation est donné, pour $e_{\mathrm{c}}=10$, par

$$
\left(\delta n / n_{\mathrm{e}}\right) t(\mathrm{ps})=8.2 \times 10^{-2}(m+2) n_{17}^{-1 / 2} T^{1 / 2}
$$

ce qui montre l'intérêt des impulsions profilées ( $m$ grand).

Dans le traitement de l'instabilité modulationnelle qui nous a conduit aux résultats ci-dessus, nous avons utilisé les équations dites de Zakharov, qui ne sont valables qu'à condition que l'excursion de l'électron dans le champ électrique de l'onde plasma électronique reste petit devant la longueur d'onde de la perturbation de densité ionique associée à l'instabilité considérée. Ceci nous donne la condition de validité suivante

$$
\frac{v_{\text {osc, plasma wave }}}{v_{\mathrm{t}}}<1.5\left(\frac{m_{\mathrm{i}}}{Z m_{\mathrm{e}}}\right)^{1 / 6} \approx 5.4
$$

ou encore,

$$
\delta n / n_{\mathrm{e}}<0.76 \times 10^{-2} T^{1 / 2} .
$$

En conjuguant (31) et (35), on vérifie que pour $e_{\mathrm{c}}=10$, quand

$$
\Phi_{14} \lambda^{2}>1.1 T^{1 / 2}
$$

la condition (35) n'est pas réalisée pour la valeur de saturation (31) calculée par la théorie standard de l'instabilité modulationnelle. Cependant pour les 
intensités suffisamment fortes pour que la condition (34) ne soit plus réalisée, des résultats récents [7] montrent que le taux de croissance $\gamma$ sature aux environs de $1.8 \omega_{\mathrm{pi}}$ où $\omega_{\mathrm{pi}}$ est la fréquence plasma ionique, $\omega_{\mathrm{pi}}^{2}=n_{\mathrm{i}} Z^{2} e^{2} / m_{\mathrm{i}} \varepsilon_{0}\left(\right.$ ici $\left.m_{\mathrm{i}} / Z m_{\mathrm{e}}=1836\right)$, puis décroît lentement quand l'amplitude des oscillations plasmas électroniques continue à augmenter.

\section{Conclusion.}

Sur la figure 1 , on a représenté les différentes valeurs de saturation obtenues dans le texte, pour le jeu de paramètres caractéristiques suivant,

$$
\begin{aligned}
\Delta n / n_{\mathrm{e}} & =0.5 \times 10^{-2} \\
T & =40 \mathrm{eV} \\
n_{\mathrm{e}} & =10^{17} \mathrm{~cm}^{-3} \\
l & =100 \mu \mathrm{m} .
\end{aligned}
$$

La courbe correspondant à l'instabilité modulationnelle a été tracée pour $e_{\mathrm{c}}=10$ à partir des résultats de la référence [7]. On observe dans cet exemple que, à bas flux $\left(\Phi \lambda^{2}<2 \times 10^{14} \mathrm{~W} \mathrm{~cm}^{-2}\right.$ $\mu \mathrm{m}^{2}$ ), c'est le désaccord linéaire de fréquence qui est la cause principale de saturation de l'onde plasma, aux flux intermédiaires $\left(2 \times 10^{14}<\Phi \lambda^{2}<4 \times\right.$ $10^{15} \mathrm{~W} \mathrm{~cm}^{-2} \mu \mathrm{m}^{2}$ ), l'instabilité modulationnelle joue le rôle prépondérant, enfin à haut flux $\left(\Phi \lambda^{2}>4 \times 10^{15} \mathrm{~W} \mathrm{~cm}^{-2} \mu \mathrm{m}^{2}\right)$ l'amplitude de l'onde plasma est essentiellement limitée par les effets relativistes, pour lesquels le calcul demanderait à être raffiné, le développement limité du terme relativiste conduisant à l'équation d'évolution (5) étant insuffisant pour les amplitudes de l'onde plasma considérées.

On remarque dans cet exemple que les collisions ne jouent aucun rôle dans la limitation de l'amplitude de l'onde, que les effets latéraux dus à la force pondéromotrice associée au rayonnement laser ou à l'onde plasma électronique ne sont susceptibles de jouer un rôle important que pour une tache focale de dimension inférieure à $100 \mu \mathrm{m}$ et pour des flux intermédiaires de l'ordre de $10^{14}$ à $10^{16} \mathrm{~W} \mathrm{~cm}^{-2} \mu \mathrm{m}^{2}$.

Les conditions d'obtention d'un plasma électronique d'amplitude suffisante sont donc :

- un bon accord de densité, $\Delta n / n_{\mathrm{e}}<10^{-2}$;

- une source laser intense,

$$
\Phi \lambda^{2}>10^{15} \mathrm{~W} \mathrm{~cm}^{-2} \mu \mathrm{m}^{2} ;
$$

- une grande tache focale.

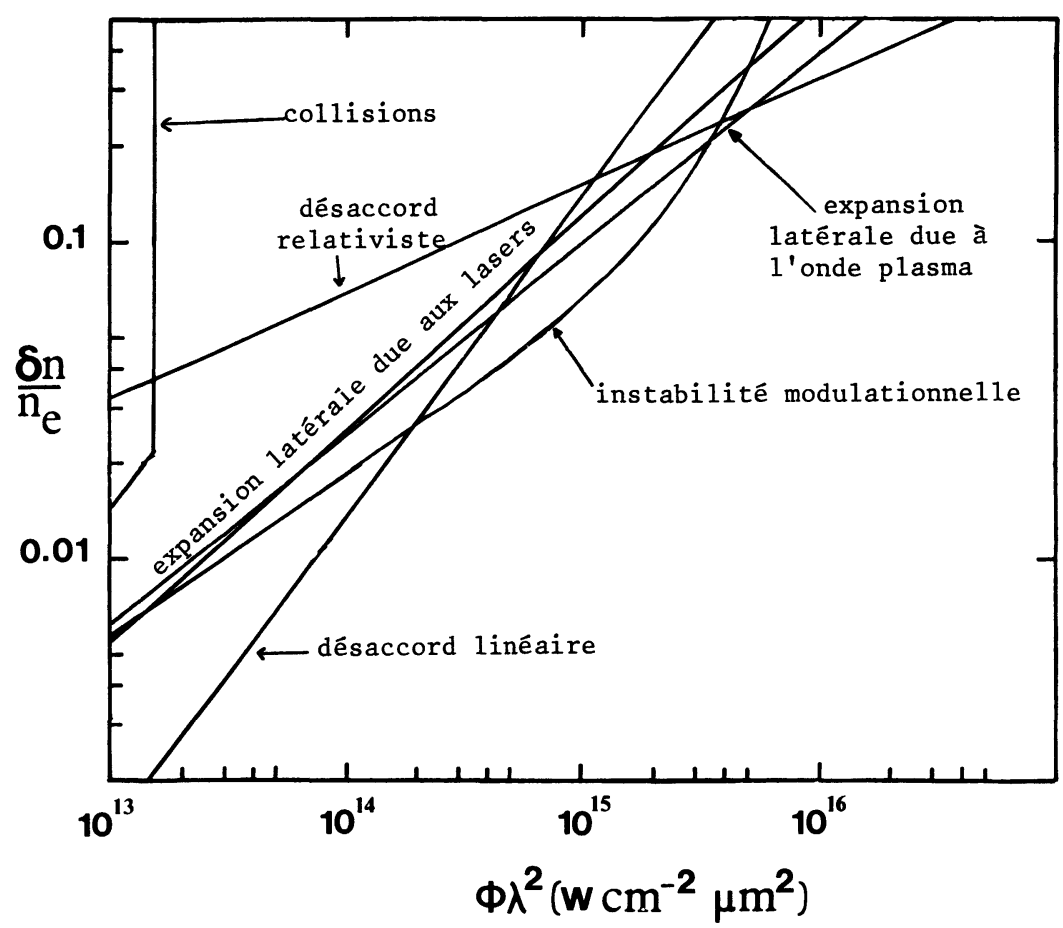

Fig. 1. - Valeurs de saturation de l'onde plasma électronique excitée par battement d'onde, dans l'hypothèse d'ondes lasers d'intensité constante, pour les différents mécanismes de saturation décrits dans le texte, considérés indépendamment les uns des autres. Les valeurs numériques des paramètres expérimentaux sont ici : $\Delta n / n_{\mathrm{e}}=0.5 \times 10^{-2}$, $T=40 \mathrm{eV}, n_{\mathrm{e}}=10^{17} \mathrm{~cm}^{-3}, l=100 \mu \mathrm{m}$.

[Saturation values of the electron plasma wave excited by the beating of two lasers, in the case of constant laser fluxes, for the different mechanisms described in the paper, considered independantly from one another. Numerical values are $\left.\Delta n / n_{\mathrm{e}}=0.5 \times 10^{-2}, T=40 \mathrm{eV}, n_{\mathrm{e}}=10^{17} \mathrm{~cm}^{-3}, l=100 \mu \mathrm{m}.\right]$ 


\section{Remerciements.}

Dans l'élaboration de ce travail, l'auteur a bénéficié de nombreuses et enrichissantes discussions avec $F$. Amiranoff, J. L. Bobin, E. Fabre, G. Coignet, C. Labaune, G. Laval, J. F. Luciani, G. Matthieussent, R. Pellat, et D. Pesme, qu'il tient à remercier ici.

\section{Annexe.}

Dans cette annexe, on détaille les effets collisionnels. On considère un plasma de charge ionique $Z=1$, et on suppose que le logarithme coulombien est égal à 4 , ce qui correspond approximativement aux gammes de densité et de température de ce papier. La fréquence de collision électron-ion $\nu_{0}$ est alors donnée par

$$
\nu_{0}=1.2 \times 10^{12} n_{17} T^{-3 / 2} .
$$

Ce résultat correspond à la limite habituelle, où la vitesse thermique électronique $v_{t}$ est beaucoup plus grande que la vitesse d'oscillation $v_{\text {osc }}$ dans le champ de l'onde laser ou dans le champ de l'onde plasma. Dans le cas général cependant, et en particulier quand $v_{\text {osc }} \geqslant v_{t}$, il est nécessaire d'inclure un facteur correctif $F$ dans l'expression de la fréquence de collision

$$
\nu=\nu_{0} F
$$

avec [8]

$$
F \approx\left(1+s^{2} / 6\right)^{-3 / 2} \quad \text { quand } \quad s^{2}<10,
$$

$$
F \approx 6 \sqrt{\frac{2}{\pi}} \frac{\ln s+\frac{1}{3}}{s^{3}} \quad \text { quand } s^{2}>10
$$

où $s$ est le rapport entre l'amplitude de la vitesse d'oscillation et la vitesse thermique,

$$
s=v_{\mathrm{osc}} / v_{\mathrm{t}}
$$

Dans le corps de l'article, on a plutôt utilisé l'approximation

$$
F \approx\left(s^{2} / 6\right)^{-3 / 2} \quad \text { pour } s \gg 1
$$

On peut définir une température équivalente $T_{\text {osc }}$ à partir de la vitesse d'oscillation $v_{\text {osc }}$

$$
T_{\mathrm{osc}}=m_{\mathrm{e}} v_{\mathrm{osc}}^{2} / 6=T s^{2} / 6 \text {. }
$$

Ainsi,

$$
T_{\text {osc, laser }}=6.3 \Phi_{14} \lambda^{2}
$$

où l'on a utilisé les normalisations données par les relations (6).

Finalement, un traitement rigoureux des effets collisionnels nécessiterait également le remplacement du logarithme coulombien utilisé dans l'équation (A.1), qui correspond à une approximation basse-fréquence $\left(\omega<\omega_{\mathrm{p}}\right)$, par le logarithme coulombien corrigé pour les effets haute-fréquence $\left(\omega \gg \omega_{p}\right)[9]$.

\section{Bibliographie}

[1] Tajima, T., Dawson, J., Phys. Rev. Lett. 43 (1979) 267.

[2] Clayton, C. E., Joshi, C., Darrow, C., UmstadTER, D., Phys. Rev. Lett. 54 (1985) 558 ;

Martin, F., Brodeur, P., Matte, J. P., Pépin, H., Ebrahim, N., S.P.I.E. Proc. 664 (1986) 20.

[3] Joshi, C., Tajima, T., Dawson, J., Baldis, H., Ebrahim, N., Phys. Rev. Lett. 47 (1981) 1285 ;

LABAUNE, C., Strasbourg 1987, Congrès National de la Société Française de Physique, Colloq. E, papier invité, à paraître Revue Phys. Appl.

[4] Bobin, J. L., Opt. Commun. 55 (1985) 413.

[5] Rosenbluth, M. N., LiU, C. S., Phys. Rev. Lett. 29 (1972) 701 ;

TANG, C. M., Sprangle, P., Sudan, R. N., Phys. Fluids 28 (1985) 1974 ;

MCKinstrie, C. J., Forslund, D. W., Phys. Fluids 30 (1987) 904.
[6] Silin, V. P., Zh. Eksp. Teor. Fiz. 48 (1965) 1679 ; Sov. Phys. JETP 21 (1965) 1127 ;

ZAKHAROV, V. E., Zh. Eksp. Teor. Fiz. 62 (1972) 1745 ; Sov. Phys. JETP 35 (1972) 908 ;

Pellat, R., Laser Plasma Interactions (Les Houches 1980), Eds. R. Balian et J. C. Adam (NorthHolland, Amsterdam) 1982, p. 411 ;

PESME, D., et. al., à paraître.

[7] MORA, P., à paraître.

[8] Silin, V. P., Zh. Eksp. Teor. Fiz. 47 (1964) 2254 ; Sov. Phys. JETP 20 (1965) 1510 ;

Shima, Y., Yatom, H., Phys. Rev. A 12 (1975) 2106 ;

Schlessinger, L., Wright, J., Phys. Rev. A 20 (1979) 1934.

[9] Dawson, J., Oberman, C., Phys. Fluids 5 (1962) 517 ;

Salat, A., Kaw, P. K., Phys. Fluids 12 (1969) 342. 